\title{
Use of IBASPM Atlas-based Automatic Segmentation Toolbox in Pathological Brains: Effect of Template Selection
}

\author{
Verónica García-Vázquez, Student Member, IEEE, Santiago Reig, Joost Janssen, Javier Pascau, Member, IEEE, \\ Alexia Rodríguez-Ruano, Ángel Udías, Judit Chamorro, Juan José Vaquero, Senior Member, IEEE, Manuel Desco
}

\begin{abstract}
IBASPM software is an atlas-based method for automatic segmentation of brain structures, available as a freeware toolbox for the SPM package. To test the influence of the atlas when segmenting normal and pathologic brains, manual segmentation of the caudate nucleus head was compared to automatic segmentations using four different atlases: the default $M N I A A L$ atlas; a customized atlas created from a combined sample of patients $(n=20)$ and controls $(n=18)$; and a customized atlas obtained separately for each group. Maximum average ratio of overlapping voxels (dice overlap) between manual and automatic segmentation was $71 \%$ for controls and $52 \%$ for patients. In both groups, overlap ratios were better when using the customized atlases, instead of the standard MNI AAL atlas. Accuracy of the method was biased between left and right hemispheres, and also between groups, individual variability being higher in patients than in controls. Volumetric measurements using the customized atlases were also more accurate than using the $M N I A A L$ atlas. Volume data were closer to manual segmentation values than dice overlap ratio (average differences ranging from $22.7 \%$ for $M N I A A L$ atlas to $10.1 \%$ for customized atlas of patients and controls combined). Results suggests a low overall performance of IBASPM as an automatic segmentation method for the head of the caudate nucleus. Because of the biases observed, the use of this method for analyzing caudate nucleus in patients presenting anatomical abnormalities should be cautiously carried out.
\end{abstract}

\section{INTRODUCTION}

CEGMENTATION of brain structures is an essential step for $N_{\text {morphometric analysis in psychiatric and neurological }}$ disorders. A variety of methods have been recently developed for segmenting regions of interest from magnetic resonance imaging (MRI) brain studies, such as edge or region detection, active shape models or snakes [1]. The Individual Brain Atlas using Statistical Parametric Mapping (IBASPM; Cuban Neuroscience Center) software [2] utilizes an atlas registration approach and has the advantage of being easily available as a

Manuscript received November 14,2008 . This work is partially funded by the following projects: CD-TEAM Project, CENIT Program (Spanish Ministerio de Industria); FIS PI052271 (Spanish Ministerio de Sanidad y Consumo); and Fundación Mutua Madrileña

V. García-Vázquez, S. Reig, Joost Janssen, Javier Pascau, Alexia Rodríguez-Ruano, Judit Chamorro, Juan José Vaquero and Manuel Desco are with Unidad de Medicina y Cirugía Experimental, Hospital General Universitario Gregorio Marañón, CIBERSAM, Madrid, 28088 Spain (desco@mce.hggm.es)

A. Udías is with Departamento de Estadística e Investigación Operativa, Universidad Rey Juan Carlos, Fuenlabrada, 28943 Spain freeware toolbox for the SPM package, a widely used tool in neuroimaging research.

The IBASPM segmentation process is based on the registration of the $M R I$ brain image to an anatomical template labeled with an anatomical atlas. The resulting transformation is reversed to map the atlas structures to the original brain geometry, thus enabling morphometric quantification. This step is performed on the gray matter segmented from the individual images using SPM segmentation.

This toolkit allows selecting both the anatomical template used for registration and the atlas used as a template for segmentation. Customized atlases can also be created following the IBASPM procedure, upon a specific group of labeled brains by estimating the likelihood of each structure [2].

The Cuban Neuroscience Center validated its segmentation with real datasets of healthy brains, based on volumetric measurements [2] and using default parameters: ICBM $152 \mathrm{Tl}$ registration template in Montreal Neurological Institute (MNI) and its corresponding labeled atlas $M N I A A L$ [4]. This validation found variable results depending on the structure, as compared to ANIMAL+INSECT atlas-based segmentation (average difference of about $11.3 \%$ for hippocampus and $41.2 \%$ for precentral sulcus)[3].

A recent work [5] compared a manual segmentation and two different approaches for automatic segmentation, IBASPM and the probabilistic-based automatic labeling FreeSurfer, using patients with chronic major depressive disorder and healthy subjects also with default parameters. Although hippocampal atrophy was detected in patients with the automatic method, the authors pointed out that manual hippocampal segmentation is still the gold standard and automatic processes need to be improved.

A major problem of atlas-based automatic segmentation techniques of pathologic brains derives from the spatial normalization to a template representing normal healthy brains, that can yield inaccurate results because of possible anatomical deviations from normality [6]. Besides, the $M N I$ $A A L$ atlas is based on a single subject, showing more anatomical detail but less inter-subject variability. The issue of template selection was studied in [7], where ten different pairs of templates (registration and atlas) were compared. The study concluded that volumetric estimations were more reliable if a 
specific pair for each subject was used instead of a single one for all subjects.

The purpose of our work was to test the accuracy of $I B A S P M$ for the segmentation of the head of the caudate nuclei in brains showing anatomical abnormalities affecting the size, shape and position of this structure. To test the impact of the atlas we compared against a manual segmentation the results provided by four different atlases: the MNI $A A L$; a customized atlas from the combined sample of patients and controls; and a customized atlas specific for each group. A sample of schizophrenic patients and a matched group of controls were included in the study.

\section{MATERIAL AND METHODS}

\section{A. IBASPM}

IBASPM relies on SPM routines for normalization (registration) and gray matter segmentation. We used SPM2 and the toolbox IBASPM downloaded from http://www.thomaskoenig.ch/Lester/ibaspm.htm in March 2007. Apart from the choice of the reference atlas, default parameters were selected in both IBASPM and SPM.

\section{B. Subjects}

The sample included 20 schizophrenic subjects (13 males) and 18 healthy controls (10 males). Mean age was 36.5 $(\mathrm{SD}=12.3)$ and $33.7(\mathrm{SD}=9.4)$, respectively, for patients and controls. The schizophrenic patients were diagnosed according to $D S M-I V$ criteria, confirmed by a semistructured interview. They were chronic patients of poor medical prognosis and thus likely to show structural alterations due to the disease. See Reig et al [6] for further details.

\section{MRI acquisition}

MRI studies were acquired on a Philips Gyroscan 1.5T scanner using a gradient echo T1-weighted 3D sequence with the following parameters: matrix size $256 \times 256$, pixel size $0.9 \times 0.9 \mathrm{~mm}$, slice thickness $1.1 \mathrm{~mm}$, flip angle $30^{\circ}$ and echo time $4.6 \mathrm{~ms}$.

\section{Manual Segmentation}

Segmentation of the caudate nuclei head was performed by manual tracing, following the protocol described in Arango et al [8]. A single operator performed the manual segmentation, thus avoiding inter-rater variability [6], [8] to facilitate comparisons.

\section{E. Evaluation}

Spatial correspondence between automatic and manual segmentation was calculated by dice overlap ratio, defined as the ratio of overlapping voxels to the total count of segmented voxels (automatic and manual segmentations combined).

Volume measurements were also obtained for each subject for comparison, considering manual segmentation as the gold standard.

\section{RESULTS}

Fig. 1 show an example of the results of the automatic segmentation compared to manual tracing in a patient (dice overlap ratio $=50 \%$ ).

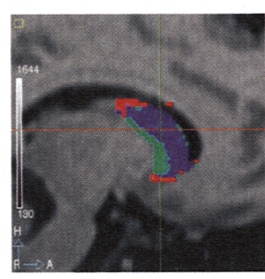

SAGITTAL VIEW

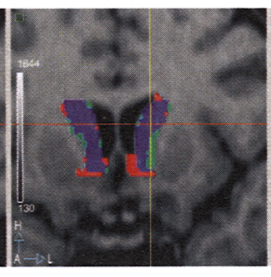

CORONAL VIEW

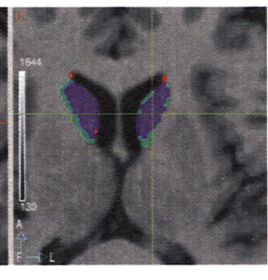

AXIAL VIEW
Fig. 1. Example of overlapping and non-overlapping pixels in a patient subject using $M N I A A L$ atlas. Red: automatic segmentation (non-overlapping voxels); purple: overlapping voxels; green: manual segmentation (nonoverlapping voxels). Images are in radiological convention: Left of the image is subject's right.

TABLE I

OVERLAP VALUES BETWEEN AUTOMATIC AND MANUAL SEGMENT ATION IN THE LEFT AND RIGHT CAUDATE NUCLEUS HEAD OF PATIENTS AND CONTROLS, USING FOUR DIFFERENT REFERENCE ATLASES (SD, STANDARD DEVIATION)

\begin{tabular}{|c|c|c|c|c|c|c|c|c|}
\hline & \multicolumn{8}{|c|}{ Atlases } \\
\hline & \multicolumn{2}{|c|}{$M N I A A L$} & \multicolumn{2}{|c|}{ All subjects } & \multicolumn{2}{|c|}{ Only Controls } & \multicolumn{2}{|c|}{ Only Patients } \\
\hline & Mean & SD & Mean & $\mathrm{SD}$ & Mean & SD & Mean & SD \\
\hline $\begin{array}{l}\text { Controls } \\
\text { (RCAUD) }\end{array}$ & 0.67 & 0.04 & $0.70^{*}$ & 0.04 & $0.71^{*}$ & 0.03 & $0.71^{*}$ & 0.03 \\
\hline $\begin{array}{l}\text { Controls } \\
\text { (LCAUD) }\end{array}$ & 0.53 & 0.09 & $0.56^{*}$ & 0.08 & $0.58^{*}$ & 0.08 & $0.58^{*}$ & 0.08 \\
\hline $\begin{array}{l}\text { Patients } \\
\text { (RCAUD) }\end{array}$ & 0.48 & 0.21 & $0.51^{*}$ & 0.20 & $0.52^{*}$ & 0.21 & $0.52^{*}$ & 0.21 \\
\hline $\begin{array}{l}\text { Patients } \\
\text { (LCAUD) }\end{array}$ & 0.36 & 0.17 & $0.38^{*}$ & 0.18 & $0.39^{*}$ & 0.18 & $0.39 *$ & 0.18 \\
\hline
\end{tabular}

(LCaud) Left Caudate Nucleus Head

(RCaud) Right Caudate Nucleus Head

${ }^{*}$ p-value $<0.01$ (Wilcoxon Signed Ranks Test between each customized atlas and the $M N I A A L$ atlas results)

Maximum average ratio of overlapping voxels (dice overlap) between manual and automatic segmentation was $\mathbf{7 1 \%}$ for controls and $52 \%$ for patients (Table I). The ratios were lower using the standard $M N I A A L$ atlas, and higher with the customized atlases. Dice overlap ratios show a high individual variability, much larger in patients than in controls. A group bias in the segmentation results was observed, yielding better results for controls than for patients. There is also a left-right bias, with better results in the right side than in the left one.

Table II shows the manual and automatic volume measurements of segmented structures (mean and standard deviation). Automatic segmentation volumes are closer to manual ones than dice overlap ratio (average differences ranged from $22.7 \%$ for $M N I A A L$ atlas to $10.1 \%$ for customized atlas using the combined sample of patients and controls). The atlas derived from the combined sample of patients and controls applied to right caudate nucleus head 
provided the minimum difference of volumetric means $(3.44$ $\mathrm{cm}^{3}$ for manual segmentation and $3.43 \mathrm{~cm}^{3}$ for that customized atlas) although the corresponding dice overlap ratio mean did not reach $80 \%$. Average volume measurements using the customized atlases were also more accurate than using the MNI $A A L$ atlas, observing similar biases as well.

TABLE II

VOLUME $\left(\mathrm{CM}^{3}\right)$ OF LEFT AND RIGHT CAUDATE NUCLEUS HEAD WITH MANUAL SEGMENTATION AND AUTOMATIC SEGMENTATION USING FOUR DIFFERENT REFERENCE ATLASES (SD, STANDARD DEVIATION)

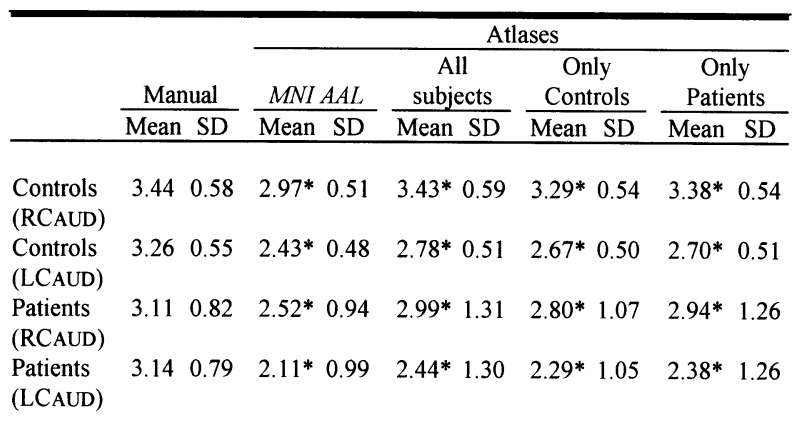

(LCaud) Left Caudate Nucleus Head

(RCaud) Right Caudate Nucleus Head

${ }^{*}$ p-value $<0.0002$ (Wilcoxon Signed Ranks Test between each

customized atlas and the MNI AAL atlas results)

\section{CONCLUSIONS}

The accuracy of IBASPM for the segmentation of the caudate nuclei head of patients presenting brain abnormalities and a matched group of controls was evaluated. Results suggests a low overall performance of IBASPM as an automatic segmentation method for the head of the caudate nucleus. Nevertheless, this process could be used as an initial step to reduce the labour of manual segmentation.

Regarding the influence of the clinical profile of the subjects, both dice overlap ratio and volumetry were better in healthy controls than in patients. This disparity could bias a comparative morphometric analyses. A critical point in the atlas-based automatic segmentation techniques and a possible cause of this difference is the registration to a template (ICBM $152 T 1$ ) representing normal healthy brains which does not properly represent inter-group variability.

The bias detected between left caudate nucleus head and right caudate nucleus head is also noticeable, thus making it advisable to perform left-right comparisons with caution.

The choice of the atlas as a template in the segmentation is another point to take into consideration. Better results were obtained using the customized atlases than the standard $M N I$ $A A L$ atlas, even for controls. The reason for this may be that these atlases better represent inter-group variability.

Finally, a good volumetric correspondence may not imply a high dice overlap ratio, as segmentation masks can be displaced, as it was observed in our sample. Although high dice overlap ratios are not necessary for volumetric analysis, adequate spatial correspondence would be essential if parameters which depend on brain region are studied as in PET images.

\section{ACKNOWLEDGMENT}

We thank Yasser Alemán-Gómez for providing information about the IBASPM toolbox.

\section{REFERENCES}

[1] M. E. Shenton, C. C. Dickey, M. Frumin,, R. W. McCarley, "A review of MRI findings in schizophrenia," Schizophr. Res., vol. 49(1-2), pp. 1$52,2001$.

[2] Y. Alemán-Gómez, L. Melie-García, and P. Valdés-Hernandez, "IBASPM: Toolbox for automatic parcellation of brain structures," Presented at the 12th Annual Meeting of the Organization for Human Brain Mapping, Florence, Italy, Available on CD-Rom in NeuroImage, vol. 27 , no. 1 , June $11-15,2006$

[3] D. L. Collins, A. P. Zijdenbos, W. F. C. Baaré, and A. C. Evans, "ANIMAL+INSECT: Improved Cortical Structure Segmentation. Information Processing in Medical Imaging," Visegrád, Editors A. Kuba, M. Sámal, A. Todd-Pokropek, vol. 1613, pp. 210-223, 1999.

[4] N. Tzourio-Mazoyer, B. Landeau, D. Papathanassiou, F. Crivello, O. Etard, N. Delcroix, et al, "Automated anatomical labelling of activations in spm using a macroscopic anatomical parcellation of the MNI MRI single subject brain," Neuroimage, vol. 15, pp.273-289, 2002

[5] W. S. Tae, S. S. Kim, K. U. Lee, E-C. Nam, and K. W. Kim, "Validation of hippocampal volumes measured using a manual method and two automated methods (FreeSurfer and IBASPM) in chronic major depressive disorder," Neuroradiology, vol. 50, no. 7, pp. 569-581, 2008.

[6] S. Reig, M. Penedo, J. D. Gispert, J. Pascau, J. Sánchez-González, P. García-Barreno and M. Desco, "Impact of ventricular enlargement on the measurement of metabolic activity in spatially normalized PET," Neuroimage, vol. 35, pp. 748-758, 2007

[7] M. Wu, C. Rosano, P. Lopez-Garcia, C. S. Carter, and H. J. Aizenstein, "Optimum template selection for atlas-based segmentation," Neurolmage, vol. 34, no. 4, pp. 1612-1618, 2007.

[8] C. Arango, A. Breier, R. McMahon, Jr. W. T. Carpenter and R. W. Buchanan, "The relationship of clozapine and haloperidol treatment response to prefrontal, hippocampal and caudate brain volumes," American Journal of Psychiatry, vol. 160, pp. 1421-1427, 2003. 